\title{
A new neutron monitor at the Juan Carlos I Spanish Antarctic Station (Livingston Island-Antarctic Peninsula)
}

\author{
J. J. Blanco*t, U. Alcalá, Spain, E-mail: juanjo.blanco@uah.es \\ O. García Población, J. I. García Tejedor, J. Medina, M. Prieto, A. López-Comazzi, S. \\ Ayuso, R. Gómez-Herrero, U. Alcalá, Spain \\ C. T. Steigies, IEAP, Christian Albrechts Universität zu Kiel, Germany
}

\begin{abstract}
A new neutron monitor was installed at Juan Carlos I Spanish Antarctic Base (S $62^{\circ} 39^{\prime} 46^{\prime \prime}$, W $60^{\circ} 23^{\prime} 20^{\prime \prime}, 12 \mathrm{~m}$ asl) last January 2019. The Base is located at Livingston Island (South Shetland Archipelago) close to the Antarctic Peninsula. The vertical rigidity cut-off for this new station is estimated as $3.52 \mathrm{GV}$. This new station (Antarctic Cosmic Ray Observatory) is composed by a BF3-based 3NM64 (ORCA) and 3 bare BF3 counters (ORCB). The neutron monitor is complemented by a muon telescope sharing a common room in a single stack. ORCA and ORCB with the Castilla-La Mancha neutron monitor (CaLMa) are the Spanish contribution to the Neutron Monitor Data Base. Juan Carlos I Base is a summer station, that that it operates only during the antarctic summer. This affects to communications and data transmission implying two different modes of data transmission, one minute resolution data and almost real time in summer and one hour resolution data that is sent once a day. Nevertheless, data with one minute resolution is stored in a NAS hard drive system along the year. First measurements and future plans are presented in this work.
\end{abstract}

36th International Cosmic Ray Conference -ICRC2019-

July 24th - August 1st, 2019

Madison, WI, U.S.A.

\footnotetext{
${ }^{*}$ Speaker.

${ }^{\dagger}$ Thanks to project CTM2016-77325-C2-1-P funded by Ministerio de Economía y Competitividad and by the European Regional Development Fund, FEDER.
} 


\section{Introduction}

A neutron monitor is a ground-based instrument designed to measure secondary neutrons produced by the interaction of cosmic rays and solar energetic particles with the atmospheric molecules [1]. Its geomagnetic location sets the energy threshold for the primary cosmic rays to be able to produce observable secondary particles at its location. A global distribution of neutron monitors transforms the Earth in a spectrometer, i.e. a detector of cosmic rays that allows to estimate the spectrum of energy of the cosmic ray flux arriving to the Earth. Particles that are monitoring by neutron monitor, around some $\mathrm{GeV}$, are strongly affected by the solar activity. This makes the neutron monitor network a global detector able to study the solar activity over a wide range of cutoff rigidities.

The Neutron Monitor Database (NMDB) is a joint effort to build, populate and maintain a centralized database of high resolution data from neutron monitor stations of all over the world [2]. This initiative was funded by the Seventh Framework Programme (FP7) of the European Commission, as a e-Infrastructure project of the Capacities Programme. The main goals are:

- Upgrade stations (acquisition systems) to they could provide real-time, high-resolution data.

- Build a centralized database with distributed database mirrors.

- Populate the database with the historical data available.

- Develop application and access tools.

- Provide public outreach information.

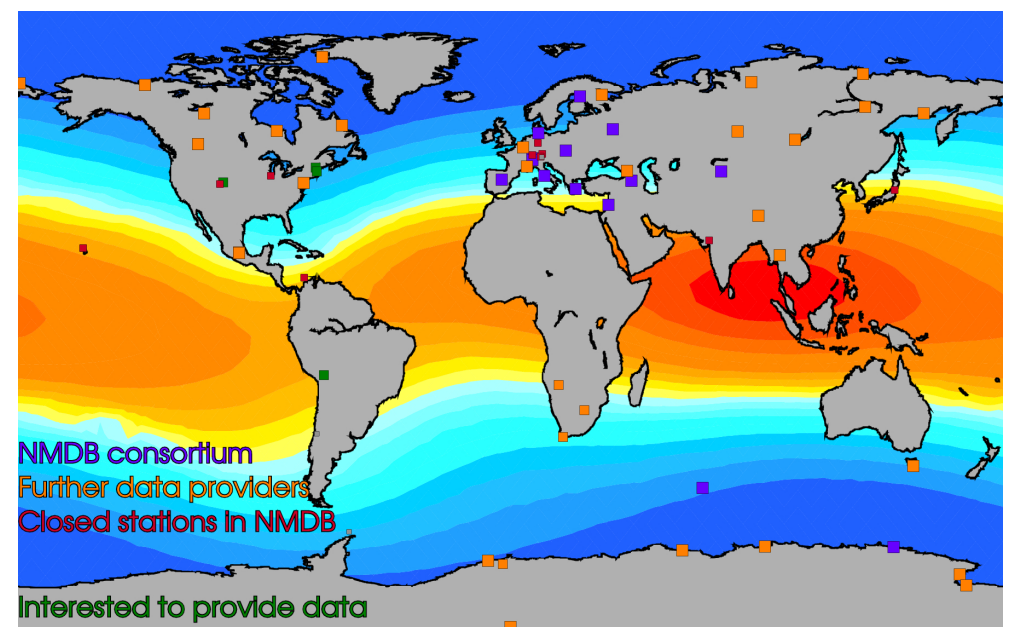

Figure 1: Neutron monitor global network.

Neutron monitors are not uniformly distributed around the World. The stations are concentrated mostly on the Northern hemisphere and Antarctica. Each neutron monitor is characterized by its altitude above sea level (asl) and vertical cut-off rigidity. The network follows the next concept; the more rigidity is covered, the more sensitive the neutron monitor network is to cosmic 
ray energy. The current neutron monitor distribution showing stations in the NMDB consortium, stations that provide data to NMDB and closed stations, is presented in figure 1.

Juan Carlos I Spanish Antarctic Base (BAE) is a summer research base located in Livingston Island, South Shetland Islands, Maritime Antarctica $62^{\circ} 39^{\prime} 46^{\prime \prime} S, 60^{\circ} 23^{\prime} 20^{\prime \prime} W$ and $12 \mathrm{~m}$ asl. The location of the base is shown in figure 2. Its location, close to the Antarctic Peninsula, covers a gap in the global distribution of neutron monitors as can be seen in figure 1. LARC neutron monitor [3] was operative at King George Island, $134 \mathrm{~km}$ apart from Livingston Island (figure 2), covering such gap in the neutron monitor global network but it is switched off nowadays. Close to the scientific module in BAE Juan Carlos I, in a thermally isolated container, the Antarctic Cosmic Ray Observatory (ORCA) was installed at the beginning of January 2019 (black container in figure $3)$.

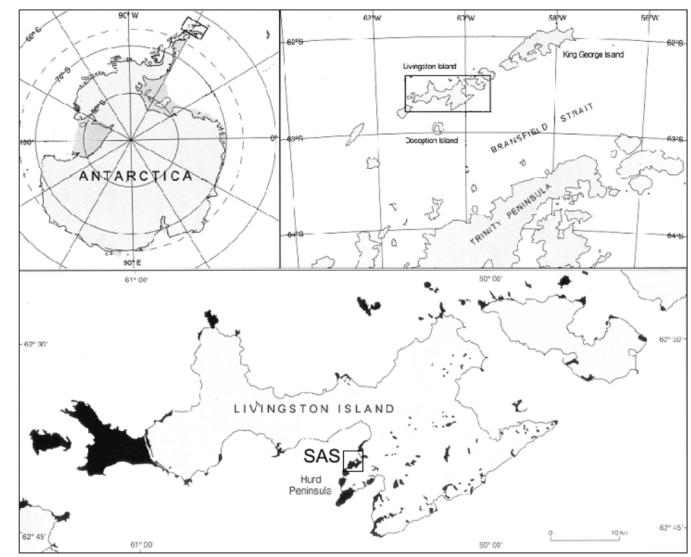

Figure 2: Map of location of Juan Carlos I Spanish Base from [4].

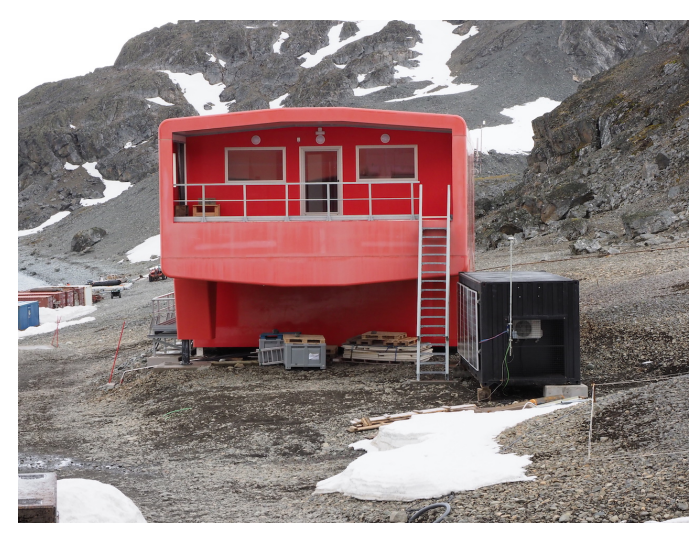

Figure 3: ORCA container and scientific module BAE Juan Carlos I.

\section{Antarctic Cosmic Ray Observatory (ORCA)}

The Antarctic Cosmic Ray observatory, ORCA, is a multi-detector (figure 4) devoted to the observation of secondary cosmic rays located at $62^{\circ} 39^{\prime} 46^{\prime \prime} S, 60^{\circ} 23^{\prime} 20^{\prime \prime} \mathrm{W}, 12 \mathrm{~m}$ asl and a vertical cut-off rigidity of $3.52 \mathrm{GV}$. It was installed at the Base after a latitudinal survey of 48 days from Vigo (Spain) to Juan Carlos I [5].

ORCA, in is current configuration, is a direct heritage of CaLMa, the Castilla-La Mancha neutron monitor located at Guadalajara (Spain) at $40^{\circ} 38^{\prime} \mathrm{N}, 3^{\circ} 9^{\prime} \mathrm{W}$ and $708 \mathrm{~m}$ above sea level [6]. It is built by a module devoted to measure neutrons (NEMO) and a muon telescope (MITO) [7] devoted to measure muons. Both modules are stacked in a common structure (figure 5). With NEMO and MITO a meteorologic station (Vaisala PTU300 Combined Pressure, Humidity and Temperature Transmitter) provides measurements of pressure, temperature and relative humidity. Data is stored in a common NAS hard drive system. NEMO, MITO and SAS are accessible via wifi and can be administered remotely. Although ORCA is connected to the Juan Carlos I power net, it also has a green power supply system composed by a set of solar panels and batteries.

ORCA started its operation on 20 January 2019 and stopped on 23 March 2019 one week after the station was closed, probably caused by the lack of power supply confirming that the system 


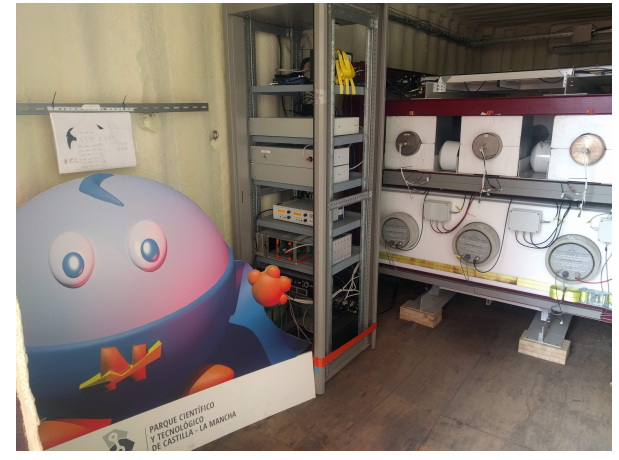

Figure 4: Picture of NEMO and MITO at Juan Carlos I Spanish Antarctic Base.

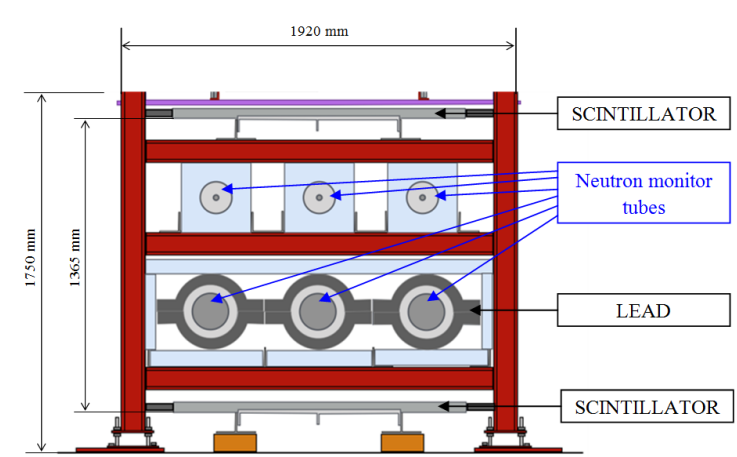

Figure 5: Layout of NEMO/MITO arrangement.

integrated by solar panels and batteries is not enough to maintain ORCA in operation. This will be checked during the next Spanish Antarctic campaign 2019-2020. During this period, NEMO was uploading data in real time to a web server. Both NEMO and MITO stored 1 min resolution data as a baseline and MITO can produce even higher time resolution because it stores particle by particle with a time resolution of $25 \mathrm{~ns}$.

NEMO is composed by a 3NM64 neutron monitor with BP28 counters and 3 bare LND2061 (3BNM) filled with $\mathrm{BF}_{3}$ at a pressure of $200 \mathrm{mmHG}$ (see figure 5). They will be named by ORCA and ORCB respectively in the NMDB. The 3BNM tubes are connected to a PDT10A-HN-12V-BF3 preamplifier. Both 3NM64 and 3BNM are controlled by an acquisition system made by three different printed circuit boards, a counter interface and power redundancy control, a Linux embedded system and a FPGA-based counter board. Details about this acquisition system can be found in [8].

NEMO measurements, i.e. secondary neutrons, are strongly affected by the air pressure, the more pressure the less counting rate, and the usable counting rate value has to be corrected taking into account this fact. The correction factor $\beta$ is obtained fitting the counting rate to a exponential law of pressure. The fits for ORCA and ORCB are presented in fig 6 obtaining $\beta_{A}=0.00735 \mathrm{hPa}^{-1}$ and $\beta_{B}=0.00639 \mathrm{hPa}^{-1}$ for ORCA and ORCB respectively. Once the factor $\beta$ is computed the counting rate can be corrected using the formula:

$$
N=N_{0} \exp \left(-\beta\left(P-P_{0}\right)\right.
$$

where $\mathrm{N}$ is the corrected counting rate, $\mathrm{N}_{0}$ is the reference counting rate, $\mathrm{P}$ is the pressure and $\mathrm{P}_{0}$ is the reference pressure.

MITO is a muon telescope made by two $1 \mathrm{~m}^{2}$ scintillator planes separated $136.5 \mathrm{~cm}$ with a lead block of $10 \mathrm{~cm}$ thickness between both (figures 4 and 5). This lead block comes from the 3NM64 (ORCA) section which is in between the scintillator planes. The generated light into the scintillator is gathered through the lateral sides thanks to four light guides. The MITO's acquisition system records the pulse high from the eight photomultiplier (PMTs) and can operate in different coincidence modes, Top, Bottom and coin8, although is possible to program it in other different coincidence modes. MITO can estimate the particle impact point on the scintillators planes by means of the comparison between the light gathered by the different PMTs. Taking into account 

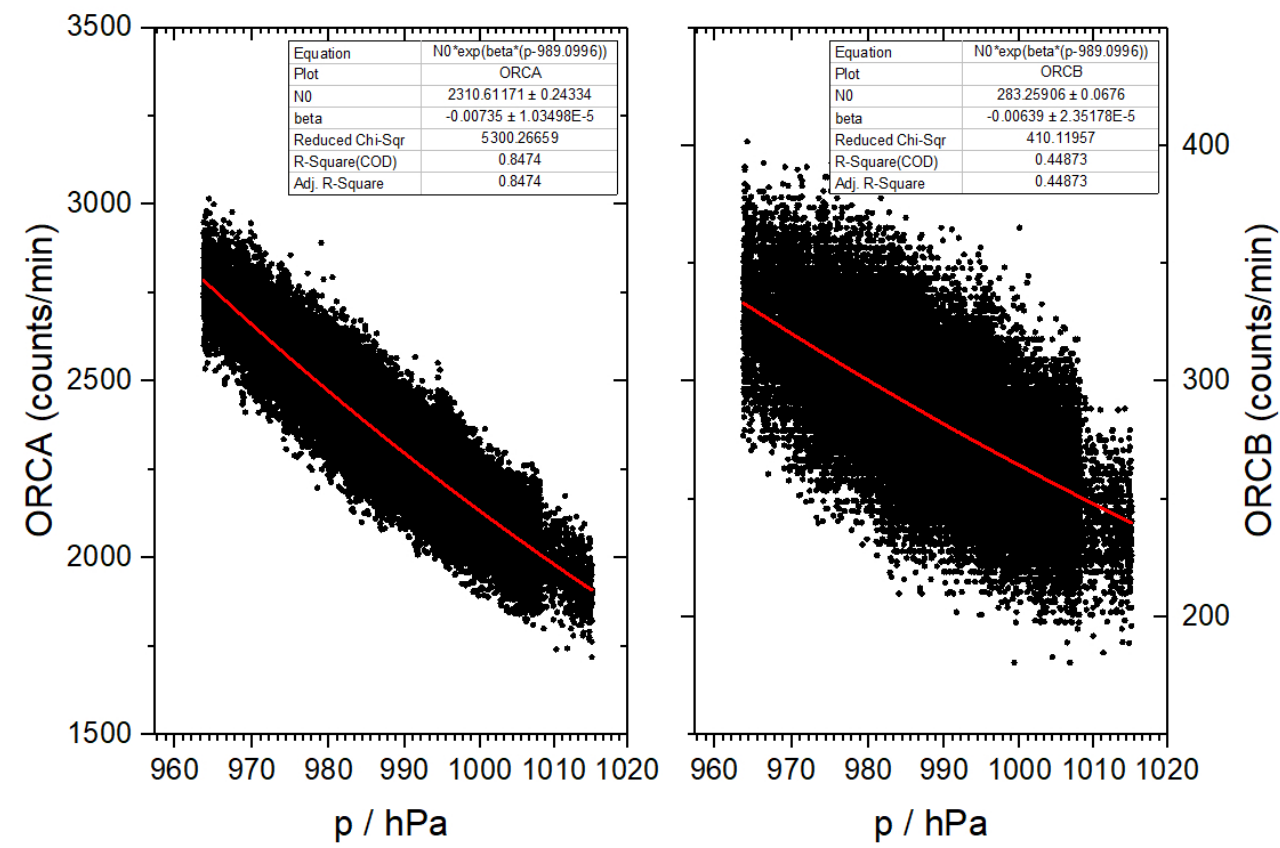

Figure 6: Counts/min vs pressure recorded from January 202019 to 23 March 2019. Red line is the fit. The fit parameters are presented in the box. Left: ORCA(3NM64). Right: ORCB(3BNM).

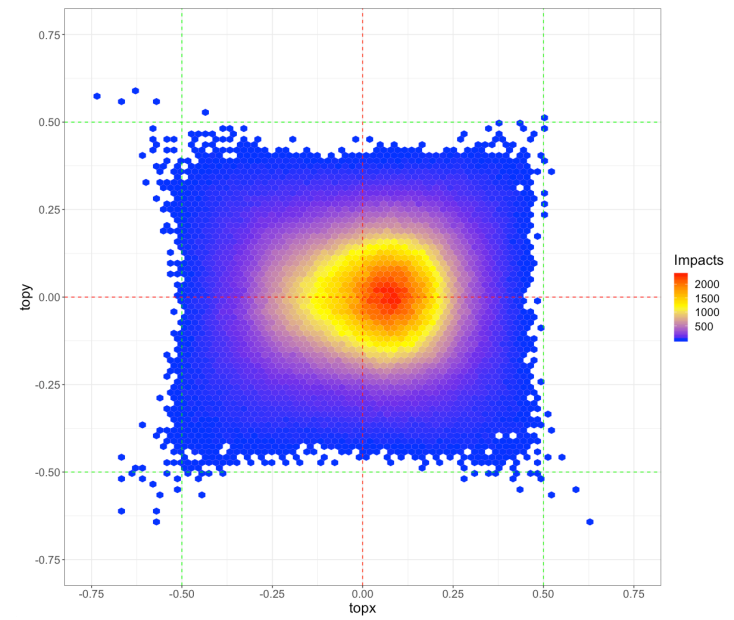

Figure 7: MITO-Top. Histogram of inferred impact points for $2^{\text {nd }}$ February 2019. $\mathrm{X}$ and $\mathrm{Y}$ axis are in meters.

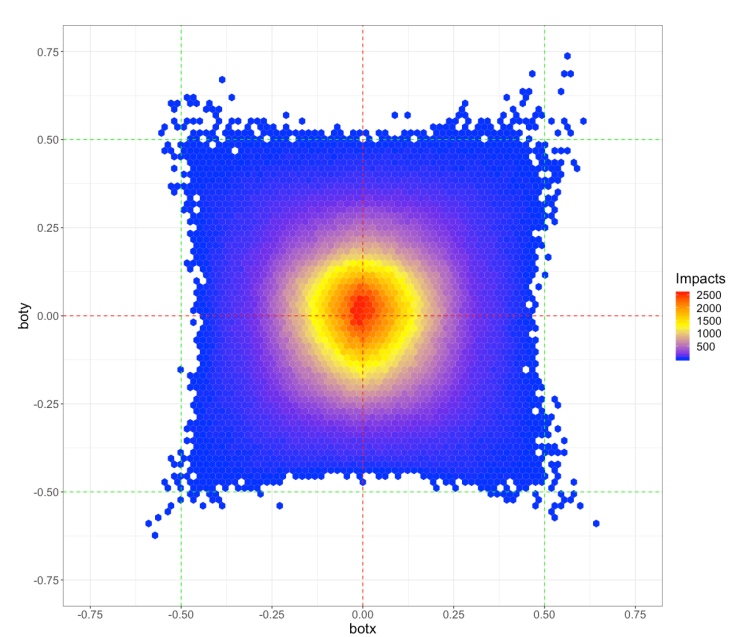

Figure 8: MITO-Bottom. Histogram of inferred impact points for $2^{\text {nd }}$ February 2019. $\mathrm{X}$ and $\mathrm{Y}$ axis are in meters. 
that the data is preliminary, an example of impact point identification is shown for the $2^{\text {nd }}$ February 2012 in figures 7 and 8.

As in the case of NEMO, MITO is also affected by the environmental conditions. Nevertheless, the pressure has a small effect on MITO (muons) when comparing with NEMO (neutrons). The obtained $\beta_{\mu}$ using equation 2.1 is $0.000921 \mathrm{ha}^{-1}$, i. e. one order of magnitude lower than $\beta_{A}$ and $\beta_{B}$. The opposite case is the dependence with temperature, both indoor and outdoor, while NEMO has a negligible temperature effect, MITO measurements have to be corrected to remove the temperature effect on muon counting rate. At the time that this proceeding is being writing this correction is a under analysis.

\section{ORCA's first light}

As it has been already commented in section 2, ORCA started its operation on 20 January 2019 and stopped to operate on 23 March 2019. During the time that BAE Juan Carlos I was operative, ORCA was measuring continuously and sending NEMO (ORCA and ORCB) data in real time, i. e. with a delay of less than 5 minutes.

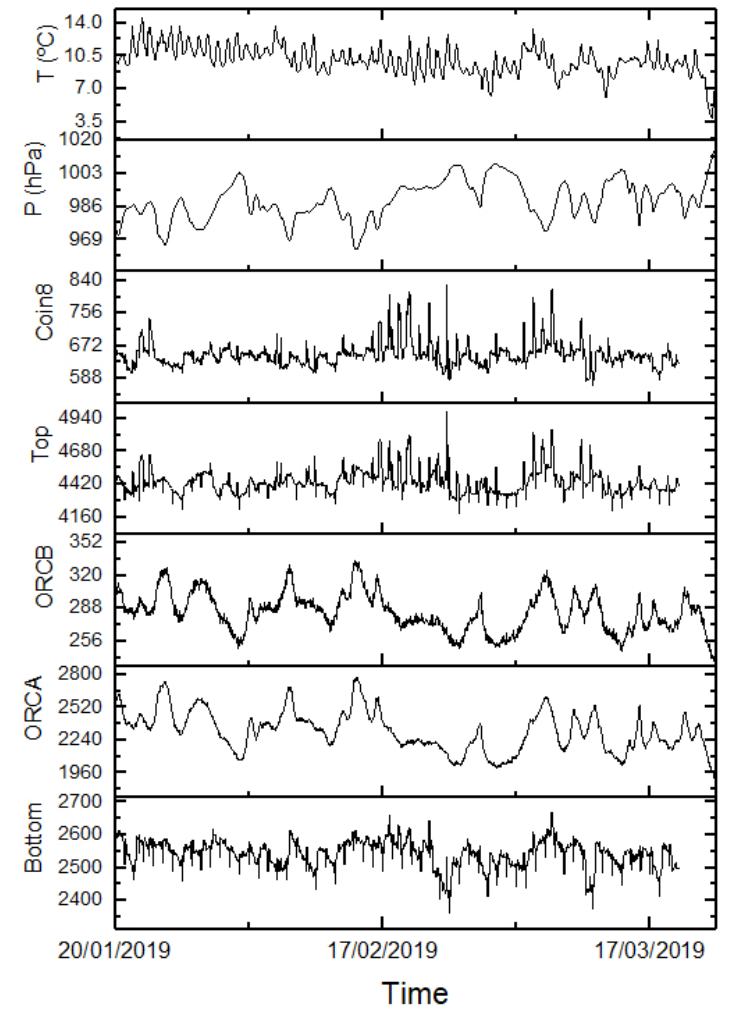

Figure 9: Main magnitudes measured by ORCA, from top to bottom: Indoor temperature in Celsius, Pressure in hPa, Coin8-MITO channel, Top-MITO channel, ORCB, ORCA and Bottom-MITO channel, all of then are given in counts/min.

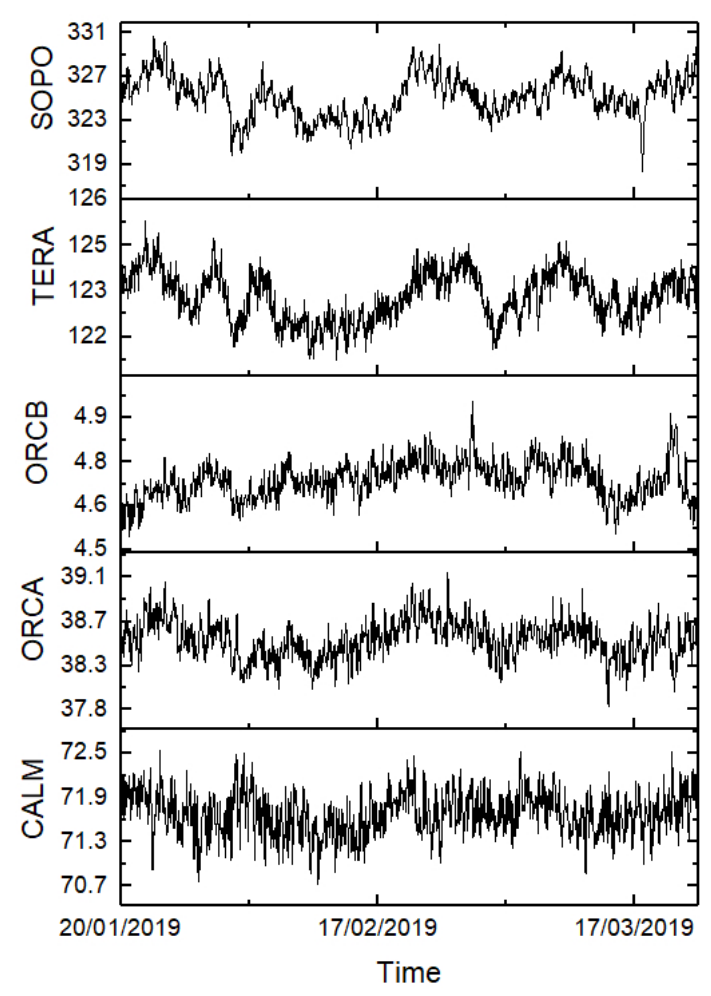

Figure 10: Neutron monitor at different rigidities and altitudes. From top to bottom: SOPO, TERA, ORCB, ORCA and CaLMa. Counting rates are pressure corrected and given in counts/s. 
As an example of the ORCA's first light, the principal observed magnitudes are plotted in figure 9. All the counting rates are uncorrected and a clear anti-correlation between the atmospheric pressure and the counting rates of ORCA and ORCB is observed. Nevertheless, the pressure effect on MITO counting rates is not so important, apparently. ORCA housing is thermally controlled maintaining an indoor temperature of about $10{ }^{\circ} \mathrm{C}$ (top panel, figure 9).

Finally, NEMO (ORCA, ORCB) pressure corrected observations have been compared with other antarctic and mid-latitude neutron monitors. The result of this comparison is shown in figure 10. From top to bottom $\mathrm{SOPO}\left(90^{\circ} \mathrm{S}, 2820 \mathrm{~m}\right.$ asl, $\left.0.1 \mathrm{GV}\right)$, TERA $\left(66.65^{\circ} \mathrm{S}, 140^{\circ} \mathrm{E}, 32 \mathrm{~m}\right.$ asl, $0.0 \mathrm{GV}), \mathrm{ORCA}$ and $\mathrm{ORCB}\left(62^{\circ} 39^{\prime} 46^{\prime \prime} \mathrm{S}, 60^{\circ} 23^{\prime} 20^{\prime \prime} \mathrm{W}, 12 \mathrm{~m}\right.$ asl, $\left.3.52 \mathrm{GV}\right)$ and CaLMa (CALM) $\left(40^{\circ} 38^{\prime} \mathrm{N}, 3^{\circ} 9^{\prime} \mathrm{W}, 708 \mathrm{~m}\right.$ asl, $\left.6.95 \mathrm{GV}\right)$. From this figure can be seen that ORCA (3NM64 section) shows similar behavior as the other antarctic stations but the similarity is not clear when comparing to CaLMa. This can be expected because during these two months the solar activity was extremely low, and under these conditions, regional effects on the magnetosphere could be more visible than global one on the neutron monitor counting rates.

Regarding ORCB (3BNM), it shows similar behavior than ORCA what it is expected although the energy threshold for neutrons detected by ORCB is lower than ORCA and that could produce some differences.

\section{Conclusions}

At the beginning of 2019 a new cosmic ray detector was installed in the Juan Carlos I Spanish Antarctic Base $\left(62^{\circ} 39^{\prime} 46^{\prime \prime} \mathrm{S}, 60^{\circ} 23^{\prime} 20^{\prime \prime} \mathrm{W}, 12 \mathrm{~m}\right.$ asl, $\left.3.52 \mathrm{GV}\right)$. This detector ORCA is a multidetector system integrated by NEMO to measure neutron and MITO to measure muons. The main conclusions that we can achieve are:

- ORCA is still in a commissioning phase and data has to be consider as preliminary.

- NEMO (ORCA and ORCB) are giving comparable measurements to other antarctic neutron monitors.

- NEMO is able to provide real-time data during summer and could be included into the NMDB once the commissioning phase is over.

- MITO is providing one-min counting rates in several coincidence channels being able to give information about the particle entrance direction on the detector volume.

\section{Acknowledgements}

Data of neutron monitors has been downloaded from NMDB page: http://www . nmolb. eu/nest/. The neutron monitor data from South Pole are provided by the University of Wisconsin, River Falls. Terre Adelie neutron monitor data were kindly provided by Observatoire de Paris and the French polar institute (IPEV), France. CaLMa neutron monitor data were kindly provided by the Space Research Group (SRG-UAH), University of Alcala, Spain. This work has been supported by the project CTM2016-77325-C2-1-P funded by Ministerio de Economía y Competitividad and by the European Regional Development Fund, FEDER. 


\section{References}

[1] John A. Simpson. The cosmic ray nucleonic component: The invention and scientific uses of the neutron monitor - (keynote lecture). Space Science Reviews, 93(1):11-32, Jul 2000.

[2] H. Mavromichalaki, A. Papaioannou, C. Plainaki, C. Sarlanis, G. Souvatzoglou, M. Gerontidou, M. Papailiou, E. Eroshenko, A. Belov, V. Yanke, E.O. Flückiger, R. Bütikofer, M. Parisi, M. Storini, K.-L. Klein, N. Fuller, C.T. Steigies, O.M. Rother, B. Heber, R.F. Wimmer-Schweingruber, K. Kudela, I. Strharsky, R. Langer, I. Usoskin, A. Ibragimov, A. Chilingaryan, G. Hovsepyan, A. Reymers, A. Yeghikyan, O. Kryakunova, E. Dryn, N. Nikolayevskiy, L. Dorman, and L. Pustil'Nik. Applications and usage of the real-time neutron monitor database. Advances in Space Research, 47(12):2210 2222, 2011. Recent Advances in Space Weather Monitoring, Modelling, and Forecasting - 2.

[3] E.G. Cordaro, E. Olivares, D. Galvez, D. Salazar-Aravena, and D. Laroze. New ${ }^{3}$ He neutron monitor for chilean cosmic-ray observatories from the altiplanic zone to the antarctic zone. Advances in Space Research, 49(12):1670 - 1683, 2012.

[4] Christian Hauck, Goncalo Vieira, Stephan Gruber, Juanjo Blanco, and Miguel Ramos. Geophysical identification of permafrost in livingston island, maritime antarctica. Journal of Geophysical Research: Earth Surface, 112(F2), 2007.

[5] J.J. Blanco, O. García Población, J. I. García Tejedor, C. T. Steigies, J. Medina, M. Prieto, A. López-Comazzi, S. Ayuso, R. Gómez-Herrero, J. A. Garzón, D. García-Castro, P. Cabanelas, A. Gomis-Moreno, V. Villasante-Marcos, B. Heber, A. Morozova, G. Kornakov, T. Kurtukian, A. Blanco, L. Lopes, J.P. Saravia, H. Kruger, Du T. Strauss, and V. G. Yanke. ORCA 2018 latitudinal survey. POS ( ICRC2019) 1059. In P. Desiati, T. Gaisser, and A Karle, editors, 36th International Cosmic Ray Conference (ICRC2019), volume 1 of Proceedings of Science, July 2019.

[6] J. Medina, J. J. Blanco, O. García, R. Gómez-Herrero, E. J. Catalán, I. García, M. A. Hidalgo, D. Meziat, M. Prieto, J. Rodríguez-Pacheco, and S. Sánchez. Castilla-La Mancha neutron monitor. Nuclear Instruments and Methods in Physics Research A, 727:97-103, November 2013.

[7] S. Ayuso, J.I. García Tejedor, J.J. Blanco, R. Gómez-Herrero, J. Medina, and O. García Población. MITO, a new directional muon telescope design. First observations. PoS ( ICRC2019) 180. In P. Desiati, T. Gaisser, and A Karle, editors, 36th International Cosmic Ray Conference (ICRC2019), volume 1 of Proceedings of Science, July 2019.

[8] Ó. G. Población, J. J. Blanco, R. Gómez-Herrero, C. T. Steigies, J. Medina, I. G. Tejedor, and S. Sánchez. Embedded data acquisition system for neutron monitors. Journal of Instrumentation, 9(08):T08002-T08002, aug 2014. 